\title{
Characterization of XANES spectra for olivine group minerals as a function of orientation and composition
}

Nichole R. Valdez, Mickey E. Gunter, M. Darby Dyar

XANES spectra have been shown to be dependent on crystal orientation. This study characterizes the variation in spectra along the $a, b$, and c crystallographic axes for the orthorhombic olivine group minerals of varying compositions where $\mathrm{Mg}_{\mathrm{x}} \mathrm{Fe}_{1-\mathrm{x}} \mathrm{SiO} 4$. Single crystal X-ray diffraction was used to collect the unit cell parameters, atomic coordinates, and bond lengths. The same single crystals were then oriented optically using a spindle stage for Xray absorption spectroscopy, and for some crystals, the composition was determined by electron microprobe analysis afterward. The compositions from refinement were compared to electron microprobe results from analysis of the bulk mineral samples, or the same single crystal when possible, and are nearly identical. The partitioning of $\mathrm{Mg}$ and Fe into the M1 and M2 crystallographic sites was found to be correlated to the total $\% \mathrm{Mg}$ in each sample. The three crystallographic axes are visually distinct in the XANES region; however samples with lower iron are more easily distinguishable, possibly due to self-absorption. There is a correlation at the pre-edge between peak intensity and higher total iron, and $\% \mathrm{Fe}^{3+}$ does not influence this trend. Ideas for the expansion of the project into other space groups are presented with preliminary results from different minerals.

Nichole R. Valdez University of Idaho

Mickey E. Gunter University of Idaho

M. Darby Dyar Mount Holyoke College 